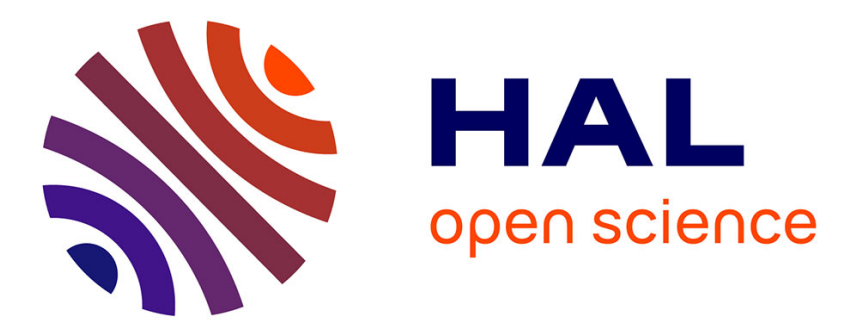

\title{
4-mecanum wheeled mobile robot actuator fault detection \& isolation using unknown input observer-based approach
}

Samia Mellah, Guillaume Graton, El Mostafa El Adel, Mustapha Ouladsine, Alain Planchais

\section{To cite this version:}

Samia Mellah, Guillaume Graton, El Mostafa El Adel, Mustapha Ouladsine, Alain Planchais. 4mecanum wheeled mobile robot actuator fault detection \& isolation using unknown input observerbased approach. European Control Conference ECC, May 2020, Saint Petersbourg, Russia. hal$02454947 \mathrm{v} 2$

\section{HAL Id: hal-02454947 \\ https://hal.science/hal-02454947v2}

Submitted on 20 Feb 2020

HAL is a multi-disciplinary open access archive for the deposit and dissemination of scientific research documents, whether they are published or not. The documents may come from teaching and research institutions in France or abroad, or from public or private research centers.
L'archive ouverte pluridisciplinaire $\mathbf{H A L}$, est destinée au dépôt et à la diffusion de documents scientifiques de niveau recherche, publiés ou non, émanant des établissements d'enseignement et de recherche français ou étrangers, des laboratoires publics ou privés. 


\title{
4-mecanum wheeled mobile robot actuator fault detection $\&$ isolation using unknown input observer-based approach
}

\author{
Samia MELLAH ${ }^{1}$, Guillaume GRATON ${ }^{1,2}$, El Mostafa EL ADEL ${ }^{1}$, Mustapha OULADSINE ${ }^{1}$ and \\ Alain PLANCHAIS ${ }^{3}$
}

\begin{abstract}
This paper proposes an approach for actuator fault detection $\&$ isolation (FDI) in a four mecanum wheeled mobile robots (4-MWMRs). The approach is based on a bank of unknown input observers (UIO) for linear parameter varying (LPV) systems. The FDI is challenging by considering faults with small amplitude and measurements with noise. Added to that, and considering the robot closed-loop control, the faults are compensated and they cannot be detected without a robust FDI algorithm. The objective is to detect and isolate actuator faults before the robot closed-loop deteriorates and leads to an unacceptable extent.
\end{abstract}

keywords: 4-mecanum wheeled mobile robot, dynamic model, actuator faults.

\section{INTRODUCTION}

Mobile robots, thanks to their capability of replacing humans to achieve hard and repetitive tasks of handling and transportation, become a subject of high interest in the industrial application domain as well as in the scientific research area.

Mainly, there is two different classes of mobile robots. A robot is called "nonholonomic" when the number of its controllable degrees of freedom (DOFs) is less than the total DOFs. For example, unicycle and car-like mobile robots are limited to only two DOFs in a planar surface. It is called "holomic" when the number of its controllable DOFs is equal to the total DOFs, e.g. omnidirectional robots have three DOFs in a planar surface (i.e, translation along $x$ and $y$ axes and rotation along $z$ axis of the inertial frame) [1].

Omnidirectionality is the ability of reaching any configuration from any initial position without having to change the orientation angle. This property is obtained thanks to the omnidirectional wheels structure.

The concept of omnidirectional wheels is based on a central wheel with free rollers mounted at an angle around the periphery of the wheel. Depending on the rollers type and their inclination angle, several types of omnidirectional wheels are distinguished [2], [3]. "Mecanum wheels" is one of them with spherical rollers placed at an angle of $45^{\circ}$ to the wheel hub circumference (see Fig. 1). More details can

\footnotetext{
*Research leading to these results has received funding from the EU ECSEL Joint Undertaking under grant agreement n 737459 (project Productive4.0) and from the partners national funding authorities DGE

${ }^{1}$ S. Mellah, G. Graton, E. M. El Adel and M. Ouladsine are with Aix Marseille Univ, Université de Toulon, CNRS, LIS (UMR 7020), Avenue Escadrille Normandie-Niemen, F-13397 Marseille Cedex 20, France samia.mellah@lis-lab.fr

${ }^{2}$ G. Graton is with Ecole Centrale Marseille, Technopôle de ChâteauGombert, 38 rue Frédéric Joliot-Curie, F-13451 Marseille Cedex 13, France

${ }^{3}$ A. Planchais is with ST Microelectronics Rousset, 190 avenue Celestin Coq, ZI - Rousset-Peynier, F-13106 Rousset, France
}

be found in [4]. According to [5], robots with mecanum wheels are more appropriate for carrying heavy goods in the industrial environment. This work is focused on fourmecanum wheeled mobile robots (4-MWMR).

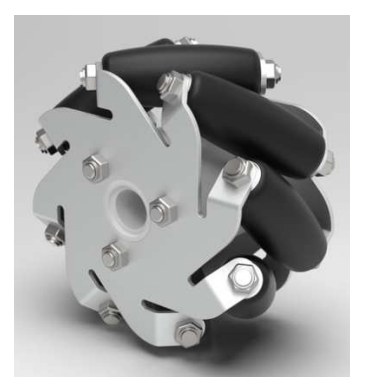

Fig. 1: Mecanum wheel design.

\section{A. Problem description}

This work is a part of the European project PRODUCTIVE4.0, with an application in STMicroeletronics, RoussetFrance, a semiconductor manufacturing company. In order to modernize its fabrication facility (fab), STMicroeletronics planes to use 4-MWMRs to transport products from an equipment to another.

Although the mobile robot ability of replacing human operators in industry applications to perform difficult and repetitive tasks quickly, efficiently, and without getting tired, they are unfortunately like all embedded systems, subject to faults.

A fault is defined as a non-permitted deviation of at least one characteristic property of a variable from an acceptable behavior [6]. Depending on the system, the presence of a fault can lead to the system performance deterioration, system instability, or even to the loss of the entire system.

According to [7], [8], a fault can be additive or multiplicative. It can be abrupt, intermittent, or even incipient [8]. Generally, faults can appear at three levels: system level, actuator level, or even sensor level. This work deals with actuator faults, where different types can be defined such as:

- Saturation: when it is highly solicited, the actuator stays stuck at the maximal value, $u_{\text {executed }}=u_{\max }$.

- Drift: actuator efficiency is deteriorated with time due to wear, $u_{\text {executed }}=u_{\text {calculated }}-\alpha$ t.

- Loss of efficiency: gain factor changed on the actuator function, $u_{\text {executed }}=\beta u_{\text {calculated }}, \beta<1$.

\section{B. Generalities}

For security reasons, it is highly important to detect and isolate autonomously the internal faults before malfunctions 
or failures. In the literature, two main classes of FDI approaches can be distinguished: data-driven methods, and model-based methods [7]. Data-based methods require a collection and manipulation of a large quantity of data, while model-based methods require generally the system physics knowledge. In our case, due to the lack of data, model-based approaches using the system mathematical model are a good compromise for FDI.

Residuals are the difference between the system calculated inputs or measured outputs and their estimations using several methods (parity-space, observers, ...) [7]. Ideally, in the absence of faults, all the residuals are equal to zero. Whereas in the presence of a fault, residuals that are sensitive to it go away from zero. In real applications, the fault is not the only reason which makes residuals different from zero because systems can be subject to disturbances and noises. Hence, the FDI algorithms should be designed to be, as less as possible, sensitive to noise and disturbances.

In this work, the robot evolves in an environment where only obstacles can disturb its predefined trajectory tracking (i.e, no wheel slippage, no friction, no slopes, ...). Moreover, obstacles are taken into account in the robot navigation. Thereby, they are not considered as disturbances. So, the robot is assumed to be subject to sensor noises only. Generally, two main steps are basically used to reduce the noise effect on residuals and deal with false alarms [7]:

- Residual filtering.

- Residual statistical testing, by imposing thresholds. Indeed, when a residual exceeds its predefined thresholds, it is tuned to be different from zero.

Thresholds can be fixed or adaptive [9]. Adaptive ones are dedicated to systems subject to uncertainties and disturbances. Therefore, fixed thresholds are used in this work.

\section{Some related works}

In [10], a survey of fault diagnosis and fault tolerant control for wheeled mobile robots (WMR) is proposed. Some model-based techniques used in WMR FDI are summarized in [11].

Many approaches are proposed to detect and isolate WMR sensor faults. In [12] a bank of Kalman filters (KF) is used. Authors in [13] propose to combine a KF bank and an expert system. In [14], a KF based on the identification technique is used to deal with the fault isolation issues.

On the other hand, WMR actuator faults, although their importance, are not widely studied. In [15], a methodology using a structural analysis-based technique is proposed to detect a unicycle mobile robot actuator faults, while in [16], authors propose to combine a KF and hardware redundancy to detect and isolate both of sensor and actuator faults in an unicycle mobile robot.

Regarding 4-MWMR, authors in [1] propose a fault tolerant control to compensate actuator faults. The proposed method seems to be efficient, but the FDI technique is not detailed. The work is based on assuming that the FDI procedure is done by a robust algorithm without giving more details. Recently, an approach based on using KF is given in
[17] to detect and isolate both of sensor and actuator faults in a 4-MWMR. The obtained results are satisfying, but only additive faults are considered and the methodology is given by assuming to have the possibility of measuring the angular velocity of all wheels adding to $x$ and $y$ positions and the rotation angle. Moreover, the robot closed-loop control is not taken into account in simulations.

\section{Main contribution}

This paper aims to detect and perfectly isolate a 4-MWRM actuator faults with only four available measurements: $x$ and $y$ positions, the rotation angle and velocity.

The FDI is challenging considering the studied fault amplitude. Taking into account the robot closed-loop, and when a small fault amplitude appears in one actuator level, it is compensated via the control law by soliciting more the other actuators. Thereby, the fault effect is not remarkable on the robot behavior. But if the latter persists, the rest of healthy actuators are highly solicited, and they risk to saturate. This can lead to the robot instability or non-controllablility, which represents a real risk for human operators in the fab.

For security reasons and for avoiding the loss of the robot while operating, any fault must be detected and isolated as soon as possible, before the robot closed-loop deteriorates to an unacceptable behavior.

Under an actuator fault, the executed input is different from its calculated input. Executed inputs estimation and their comparison with the controller outputs (calculated inputs) allows to detect when there is an abnormality. Indeed, when the comparison result (residuals) are different from zero, a fault is detected.

Taking into account the system characteristics, unknown input observer (UIO) for linear parameter varying (LPV) systems [18] is proposed in this work. The aim is to estimate actuator executed inputs, which are assumed to be unknown, and compare them with the calculated ones to detect and isolate internal faults without any human intervention. Simulation results show that this method is very promising.

The outline of this paper is as follows: in the upcoming section, the mathematical model and the closed-loop control of a 4-MWMR are given. Then, the UIO for LPV systems theory is described. Next, the residual generation principle for actuator fault FDI is exposed, and simulation results are given, followed finally by a conclusion and perspectives.

\section{4-MWMR MODEL \& CLOSED-LOOP CONTROL}

Fig. 2 shows a 4-MWMR geometry. It is assumed that the robot is placed on a plane surface where $(O, \vec{x}, \vec{y})$ is the inertial reference frame and $\left(G, \overrightarrow{x_{R}}, \overrightarrow{y_{R}}\right)$ is a local coordinate frame fixed on the robot at its center of mass $G$.

\section{A. Mathematical model}

To consider the robot dynamic model, the following assumptions are made:

- Disturbances are neglected thanks to the robot evolving environment.

- Four measurements are available: $x$ and $y$ position provided by a positioning system, rotation angle $\theta$ 


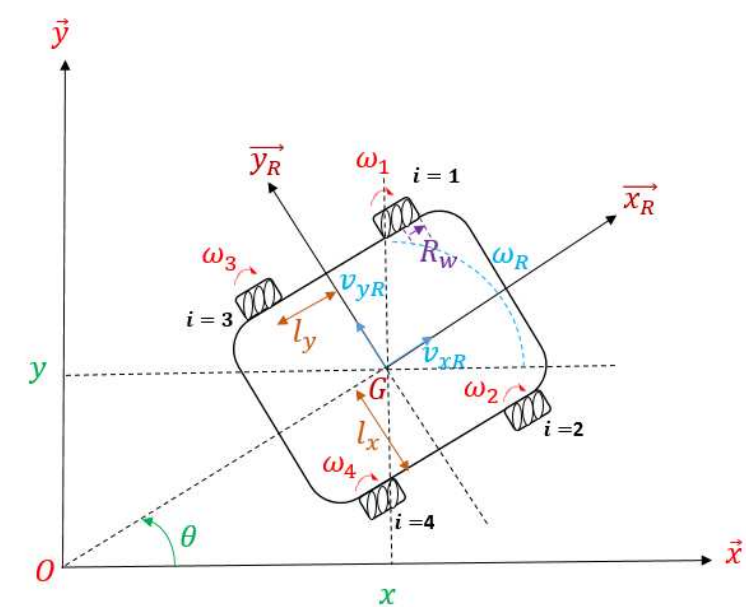

Fig. 2: Geometry of a 4-mecanum wheeled robot.

returned by a gyroscope, and the rotational velocity $\dot{\theta}$ provided by a gyrometer.

- Measurement noises are modeled by taking into account sensor accuracy.

Table I below summarizes the notations used throughout this article (see Fig. 1).

\begin{tabular}{|c|c|}
\hline Variable & Description \& unit \\
\hline$x, y$ & Robot position along $\mathrm{x}$-axes and $\mathrm{y}$-axes $(\mathrm{m})$. \\
\hline$\theta$ & Robot orientation angle (rad). \\
\hline$l_{x}$ & Half distance between front or rear wheels (m). \\
\hline & Half distance between front and rear wheels ( $\mathrm{m}$ ). \\
\hline $\begin{array}{l}l=l_{x}+l_{y} \\
R_{w}\end{array}$ & Wheel radius $(\mathrm{m})$. \\
\hline$(\dot{x}, \dot{y}),\left(\dot{x}_{R}, \dot{y}_{R}\right)$ & $\begin{array}{l}\text { Linear velocities }(\mathrm{m} / \mathrm{s}) \text { in the inertial and local } \\
\text { reference frame respectively. }\end{array}$ \\
\hline$(\ddot{x}, \ddot{y}),\left(\ddot{x}_{R}, \ddot{y}_{R}\right)$ & $\begin{array}{l}\text { Linear accelerations }\left(\mathrm{m} / \mathrm{s}^{2}\right) \text { in the inertial } \\
\text { and local reference frame respectively. }\end{array}$ \\
\hline$\dot{\theta}, \ddot{\theta}$ & Rotational velocity $(\mathrm{rad} / \mathrm{s}) /$ acceleration $\left(\mathrm{rad} / \mathrm{s}^{2}\right)$. \\
\hline$I_{z}$ & Moment of inertia of the platform $\left(\mathrm{kg} \cdot \mathrm{m}^{2}\right)$ \\
\hline$m$ & \\
\hline$\tau_{i}$ & Applied torque to each wheel $i$ (N.m). \\
\hline
\end{tabular}

TABLE I: Robot nomenclature

1) Dynamic model: Neglecting the model uncertainties and frictions, the dynamic model is given in the robot local frame by the following equations: (see [19] for more details)

$$
\left\{\begin{aligned}
\ddot{x}_{R} & =\frac{1}{2 m R_{w}}\left(\tau_{1}+\tau_{2}+\tau_{3}+\tau_{4}\right) \\
\ddot{y}_{R} & =\frac{1}{2 m R_{w}}\left(\tau_{1}-\tau_{2}+\tau_{3}-\tau_{4}\right) \\
\ddot{\theta} & =\frac{l}{2 I_{z} R_{w}}\left(\tau_{1}-\tau_{2}-\tau_{3}+\tau_{4}\right)
\end{aligned}\right.
$$

This model can be expressed in the inertial reference frame $(O, \vec{x}, \vec{y})$ using the following transformation matrix: [1]

$$
\left[\begin{array}{c}
\dot{x} \\
\dot{y} \\
\dot{\theta}
\end{array}\right]=R(\theta)\left[\begin{array}{c}
\dot{x}_{R} \\
\dot{y}_{R} \\
\dot{\theta}
\end{array}\right], R(\theta)=\left[\begin{array}{ccc}
\cos \theta & -\sin \theta & 0 \\
\sin \theta & \cos \theta & 0 \\
0 & 0 & 1
\end{array}\right]
$$

2) Continuous-state space representation: Using (1) and (2), the robot model is given as follows:

$$
\left\{\begin{array}{l}
\dot{X}=A X+B_{\theta} u \\
Y=C X+w
\end{array}\right.
$$

where $X=[x, y, \theta, \dot{x}, \dot{y}, \dot{\theta}]^{T}, u=\left[\tau_{1}, \tau_{2}, \tau_{3}, \tau_{4}\right]^{T}$ and $w$ denotes the sensor noises, assumed to be uncorrelated Gaussian white noises with known variances linked to the sensors accuracy. By considering $a=\frac{1}{2 m R_{w}}, b=\frac{1}{2 I_{z} R_{w}}$, $c=\cos \theta, d=\sin \theta$, it follows:

$$
\begin{gathered}
A=\left[\begin{array}{llllll}
0 & 0 & 0 & 1 & 0 & 0 \\
0 & 0 & 0 & 0 & 1 & 0 \\
0 & 0 & 0 & 0 & 0 & 1 \\
0 & 0 & 0 & 0 & 0 & 0 \\
0 & 0 & 0 & 0 & 0 & 0 \\
0 & 0 & 0 & 0 & 0 & 0
\end{array}\right], C=\left[\begin{array}{cccccc}
1 & 0 & 0 & 0 & 0 & 0 \\
0 & 1 & 0 & 0 & 0 & 0 \\
0 & 0 & 1 & 0 & 0 & 0 \\
0 & 0 & 0 & 0 & 0 & 1
\end{array}\right] \\
B_{\theta}=\left[\begin{array}{cccc}
0 & 0 & 0 & 0 \\
0 & 0 & 0 & 0 \\
0 & 0 & 0 & 0 \\
\frac{c-d}{a} & \frac{c+d}{a} & \frac{c-d}{a} & \frac{c+d}{a} \\
\frac{c+d}{a} & \frac{d-c}{a} & \frac{c+d}{a} & \frac{d-c}{a} \\
\frac{1}{b} & \frac{-1}{b} & \frac{-1}{b} & \frac{1}{b}
\end{array}\right]=\left[\begin{array}{c}
0_{(3 \times 4)} \\
\beta_{\theta}
\end{array}\right]
\end{gathered}
$$

\section{B. Closed-loop control}

Using a feedback linearizing control [20] to track a predefined robot trajectories, (3) can be controlled by introducing $\mathcal{Y}=\mathcal{X}=[x, y, \theta]^{T}$. The second derivative $\ddot{\mathcal{X}}$ is a linear expression of $u$ with varying parameters given by (4). By introducing $v$ as $v=\beta_{\theta} u$, it follows:

$$
\ddot{\mathcal{Y}}=\ddot{\mathcal{X}}=\beta_{\theta} u=v
$$

Then, using a pole placement method [20], the control law is given by:

$$
\left\{\begin{aligned}
v & =\sum_{i=0}^{n-1} a_{i}\left(Y_{r e f}^{i}-\mathcal{Y}^{i}\right)+a_{n} Y_{r e f}^{n} \\
u & =\beta_{\theta}^{\dagger} v
\end{aligned}\right.
$$

where $\mathcal{Y}^{i}$ corresponds to the $i^{\text {th }}$ derivative of $\left(\mathcal{Y}^{0}=\mathcal{Y}\right), Y_{\text {ref }}^{i}$ the $i^{\text {th }}$ derivative of the reference trajectory, $\beta_{\theta}^{\dagger}$ the pseudoinverse of $\beta_{\theta}$ such that $\beta_{\theta} \beta_{\theta}^{\dagger}=I$, the identity matrix. The polynomial coefficients $a_{i} \in \mathbb{R}$ are chosen such that the poles of polynomial $P$ defined as $P(s)=\sum_{i=0}^{n} a_{i} s^{i}$ are with nonpositive real parts. In this work, $n=2$.

\section{UNKNOWN INPUT OBSERVERS FOR LPV SYSTEMS APPLIED TO 4-MWMR}

In order to detect and isolate an actuator fault, a strategy is to build an observer bank by isolating the inputs one by one and considering them as an unknown inputs. From (3), $B_{\theta} u$ can be written as $\mathcal{B}_{\theta} \mathbf{u}+D_{\theta} d$, (3) becomes:

$$
\left\{\begin{aligned}
\dot{X} & =A X+\mathcal{B}_{\theta} \mathbf{u}+D_{\theta} d \\
Y & =C X+w
\end{aligned}\right.
$$

where $\mathbf{u}$ is a $(3 \times 1)$ input vector assumed to be known, $\mathcal{B}_{\theta}$ its control matrix, $d$ one of the four inputs (scalar) assumed to be unknown and $D_{\theta}$ its control vector.

Based on [18], the UIO associated to (6) is given by:

$$
\left\{\begin{aligned}
\dot{Z} & =N_{\theta} Z+G_{\theta} \mathbf{u}+H_{\theta} Y \\
\hat{X} & =Z-E_{\theta} Y \\
\hat{Y} & =C \hat{X}
\end{aligned}\right.
$$


Note that matrices $N_{\theta}, G_{\theta}, H_{\theta}$, and $E_{\theta}$ are not constant, and their variation depends on the orientation angle $\theta$. Let's define the state estimation error as follows:

$$
\begin{aligned}
e & =X-\hat{X} \\
& =X-Z+E_{\theta} Y \\
& =\left(I_{n}+E_{\theta} C\right) X-Z \\
& =P_{\theta} X-Z
\end{aligned}
$$

The time derivative of the estimation error is given by:

$$
\begin{aligned}
\dot{e}= & P_{\theta} \dot{X}+\dot{P}_{\theta} X-\dot{Z} \\
= & N_{\theta} e+\left(P_{\theta} A-N_{\theta} P_{\theta}-H_{\theta} C+\dot{P}_{\theta}\right) X \\
& +\left(P_{\theta} \mathcal{B}_{\theta}-G_{\theta}\right) \mathbf{u}+P_{\theta} D_{\theta} d
\end{aligned}
$$

The UIO exists for the system (6) if $e \rightarrow 0$ when $t \rightarrow \infty$. So, following conditions must be satisfied:

- $N_{\theta} e$ is asymptotically stable

- $P_{\theta} A-N_{\theta} P_{\theta}-H_{\theta} C+\dot{P}_{\theta}=0$

- $P_{\theta} \mathcal{B}_{\theta}-G_{\theta}=0$

- $P_{\theta} D_{\theta}=0$

Also, note that these two following necessary conditions [21] are satisfied by taking into account the four measured outputs cited previously:

- The rank condition $\operatorname{rank}\left(C D_{\theta}\right)=\operatorname{rank}\left(D_{\theta}\right)$.

- The pair $A_{1}, C$ must be observable, where $A_{1}=\left(A P_{\theta}+\dot{P}_{\theta}\right)-D_{\theta}\left[\left(C D_{\theta}\right)^{T} C D_{\theta}\right]^{-1}\left(C D_{\theta}\right)^{T} C A$

Finally, the unknown input $d$ can be estimated using the following equation [21]:

$$
\left.\hat{d}=\left(C D_{\theta}\right)^{\dagger} \dot{\hat{Y}}-C A \hat{X}-C \mathcal{B}_{\theta} \mathbf{u}\right]
$$

\section{FAULT DETECTION \& ISOLATION}

As said before, a bank of UIO is used for the FDI. Generally, there are two bank of observer structures: generalized observer structure introduced by Frank [22] and dedicated observer structure introduced by Clark [23]. In generalized observer structure, each observer has as input all the system outputs and inputs except one input. Hence, each observer is sensitive to all actuator faults except one. Whereas in dedicated observer structure, each observer inputs consist on all the system outputs with only one input. Thereby, each observer is sensitive to only one actuator fault.

\section{A. Residual generation}

In this work, a generalized observer structure is used. When a fault appears, it affects the system outputs. Thereby, using the system outputs as an input of all bank observers, the unknown inputs, corresponding to $u_{\text {executed }}$ in Fig. 3, are estimated with fault.

As shown in Fig. 3, the aim is to use Observer $_{i}(i=$ $1, \ldots, 4)$ to estimate the input $u_{i}$ which is not given in the observer input. Then, by comparing the system calculated inputs (controller output) and the estimated ones, four residuals are generated as follows:

$$
\left\{\begin{array}{l}
r_{1}=u_{1}-\hat{u}_{1} \\
r_{2}=u_{2}-\hat{u}_{2} \\
r_{3}=u_{3}-\hat{u}_{3} \\
r_{4}=u_{4}-\hat{u}_{4}
\end{array}\right.
$$

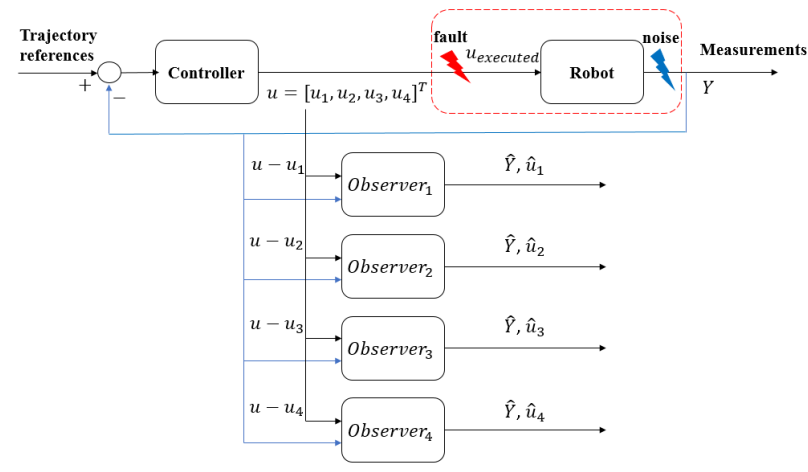

Fig. 3: Generalized observer structure.

As mentioned before, fixed thresholds are used for the fault detection. Since the sensors accuracy are known (i.e, the measurement dispersion around the real value), the thresholds are fixed using three sigma method [24].

So it is assumed that the case when a residual value is in the interval of the three sigma values, corresponds to a no-fault case. In parallel, when a residual exceeds its higher or lower predefined threshold (i.e, it leaves the healthy state values interval), a fault is detected by assigning the decision residual to 1 .

It is important to precise that by taking an interval of three sigma, only $99.97 \%$ of values are saved. The rejected ones $(0.03 \%)$ may be considered as faulty information, and hence lead to detect false alarms.

The decision algorithm must be robust against false alarms detection problem. For that, the decision residuals $R_{i}$ are turned into 1 if and only if the predefined thresholds are exceeded more than $N$ consecutive times (see (12)). The $N$ value is determined experimentally.

$$
R_{i}(k)= \begin{cases}1 & \text { if }\left(r_{i}(j)<-r_{t h, i} \text { or } r_{t h, i}<r_{i}(j)\right), \\ & \forall j \in[k-N+1, k] \\ 0 & \text { otherwise }\end{cases}
$$

where $r_{t h, i}$ is the threshold associated to residual $i$.

\section{B. Residual signature}

By taking into account the robust decision algorithm, decision residuals have the following signatures under the considered faults (see Table II below). $A_{i}$ denotes the $i_{t h}$ actuator fault.

\begin{tabular}{|c|c|c|c|c|c|}
\hline Fault & $\emptyset$ & $\mathrm{A}_{1}$ & $\mathrm{~A}_{2}$ & $\mathrm{~A}_{3}$ & $\mathrm{~A}_{4}$ \\
\hline $\mathrm{R}_{1}$ & 0 & 1 & 0 & 0 & 0 \\
\hline $\mathrm{R}_{2}$ & 0 & 0 & 1 & 0 & 0 \\
\hline $\mathrm{R}_{3}$ & 0 & 0 & 0 & 1 & 0 \\
\hline $\mathrm{R}_{4}$ & 0 & 0 & 0 & 0 & 1 \\
\hline
\end{tabular}

TABLE II: Decision residual signature under actuator faults

As seen in Table II, the fact of using a bank of UIO allows not only to detect actuator faults, but also to perfectly isolate them. 


\section{Simulation RESUlts}

The robot physical parameters are given by the following values: $m=390 \mathrm{~kg}, R_{w}=0.125 \mathrm{~m}, l_{x}=0.1825 \mathrm{~m}, l_{y}=$ $0.28 \mathrm{~m}$, and $I_{z}=50 \mathrm{~kg} \cdot \mathrm{m}^{2}$. Measurement noises take into account the sensors accuracy given by: $x$ and $y$ positions are provided by the positioning system with an error of \pm 5 $\mathrm{mm}$, while the gyrometer and the gyroscope sensors provide measures with an error of $\pm 0.5^{\circ}$ and $\pm 0.3^{\circ} / \mathrm{s}$ respectively.

As mentioned in section I.A, actuator faults can appear under three different types: saturation, loss of efficiency and drift-like faults.

In this work, the robot evolves in an environment containing human operators. So for security reasons, it moves with a low velocity. Hence, saturation faults are assumed not to happen. Therefore, only actuator loss of efficiency and drift faults are studied. In the following, simulation results for different actuator fault types and amplitudes are presented.

In Fig. 4, residuals $r_{i}$ and their thresholds are drawn to illustrate the FDI principle. In the absence of faults, all the decision residuals are set into 0 although the presence of some residual values that exceed the fixed thresholds. The decision algorithm is robust against these false alarms.
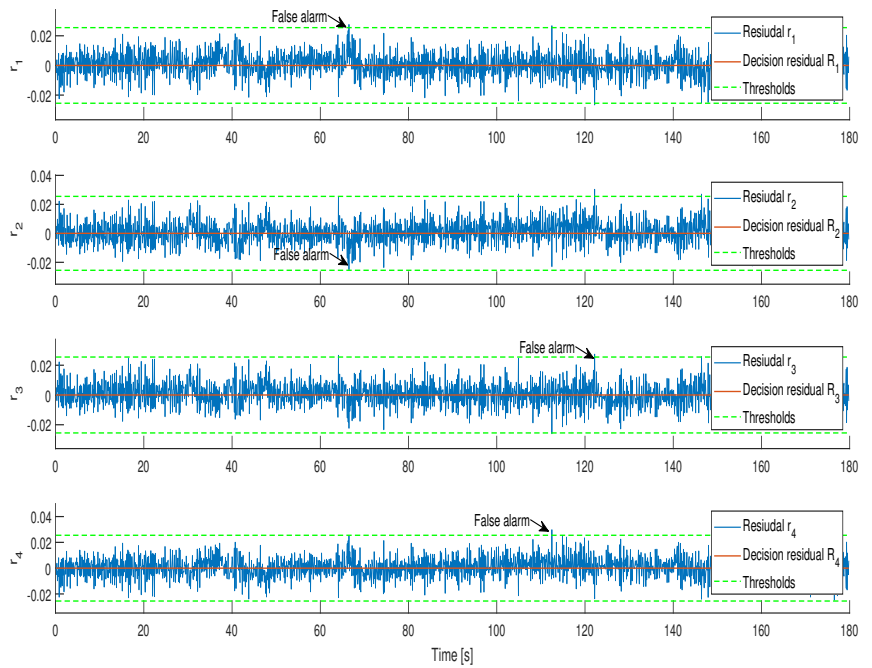

Fig. 4: Residuals in the absence of faults.

For clarification reasons, the upcoming figures contain only residuals being sensitive to the simulated faults. Other residuals are still close to zero, so they are not plotted.

Fig. 5 illustrates an FDI on the first wheel actuator (see Fig. 2). The simulated fault is a drift representing the actuator wear with respect to the time defined by: $u_{1 \text {,executed }}=u_{1}-$ $\alpha\left(t-t_{a}\right)$ (see Fig. 3), where $\alpha$ is proportional to $u_{1}$ and equals to $\alpha=2.5 e^{-5} u_{1}, t$ is the current instant and $t_{a}$ is the fault apparition instant. The fault is simulated at $6 \mathrm{~s}$ and it is detected and isolated at $13.3 \mathrm{~s}$.

In Fig. 6, a fault is simulated in the second wheel actuator level (see Fig. 2). The fault represents the second actuator loss of efficiency and it is given as follows: $u_{2, \text { executed }}=$ $(1-\beta) u_{2}, \beta=2 e^{-3}$. As seen in Fig. 6, the fault, which represents a loss of just $0.2 \%$ of the control value, is

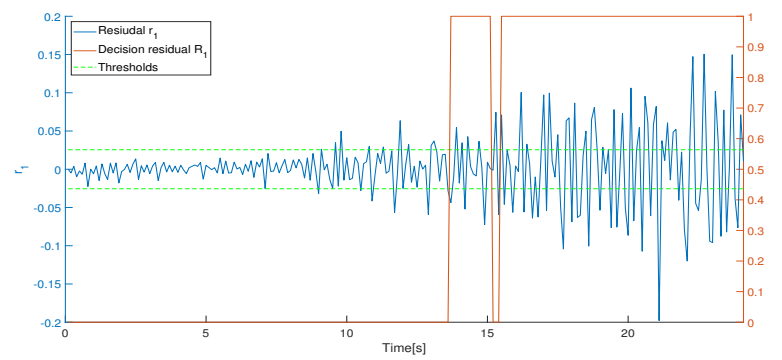

Fig. 5: Actuator 1 FDI.

simulated at the instant $36 \mathrm{~s}$ and detected \& isolated at the instant 36.6s.

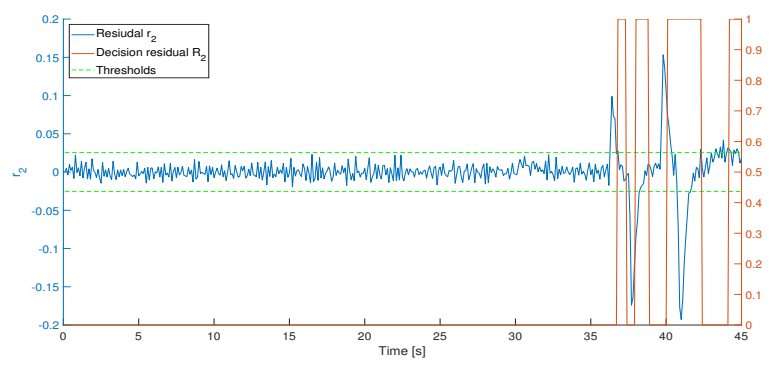

Fig. 6: Actuator 2 FDI.

Fig. 7 illustrates the detection and isolation of a fault simulated at the third wheel actuator level (see Fig. 2). The fault represents a loss of efficiency: $u_{3, \text { executed }}=(1-\gamma) u_{3}$, $\gamma=1 e^{-3}$. The latter is detected and isolated $6 s$ after its apparition, despite it represents a loss of only $0.1 \%$ of the actuator efficiency.

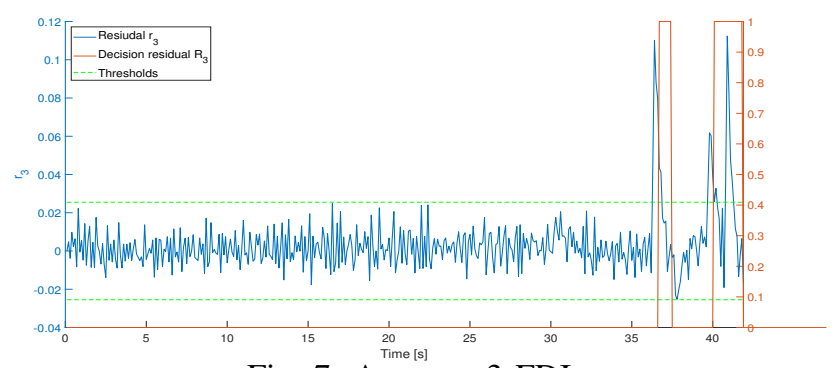

Fig. 7: Actuator 3 FDI.

Finally, in Fig. 8, a fault representing a drift of the fourth actuator is simulated. It is done as follows: $u_{4, \text { executed }}=$ $u_{4}-\zeta\left(t-t_{b}\right), \zeta=1 e^{-4} u_{4}, t$ is the current time and $t_{b}$ the fault apparition instant. The fault is simulated at $30 \mathrm{~s}$ and the FDI is done at $31 s$.

Note that in the simulated results, sometimes the fault is detected then not despite its permanent presence, then detected again. This is due to the fact that the simulated faults are proportional to the actuator control values. Hence, when an actuator is not solicited, its fault can not be seen despite its permanent presence.

\section{CONCLUSion \& PERSPECTIVES}

In this paper, a fault detection \& isolation (FDI) problem regarding actuator faults in a four mecanum wheeled mobile 


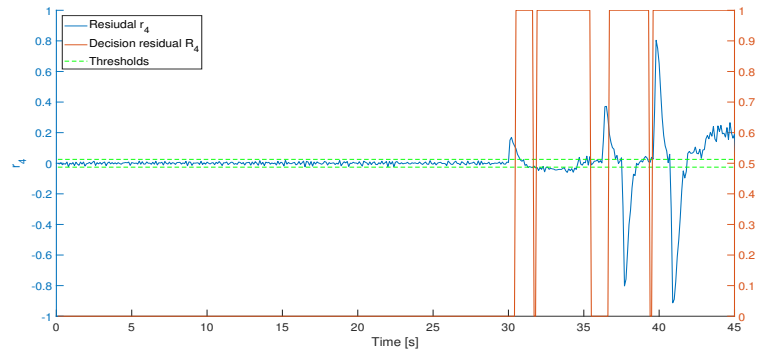

Fig. 8: Actuator 4 FDI.

robot (4-MWMR) is studied. The proposed approach is based on using a bank of unknown input observers (UIO) for linear parameter varying (LPV) systems. The FDI is challenging regarding the studied fault amplitude and measurement noises. Since these small amplitude faults are compensated by the robot closed-loop control, they cannot be seen without a robust FDI approach. The closed-loop compensation is based on soliciting more the not faulty actuators when a fault appears in one actuator. This presents a high risk when the fault persists and the healthy actuators are saturated. In this case, the closed-loop control performance will deteriorate causing the robot instability. The proposed approach is very efficient. Despite the presence of measurement noises, and the faults small amplitude, the latter are detected and isolated just few seconds after their apparition. The simulation results are very satisfying. It is planed to apply the approach on a real 4-MWMR to validate it experimentally.

\section{REFERENCES}

[1] P. Vlantis et al. "Fault tolerant control for omnidirectional mobile platforms with 4 mecanum wheels". In: IEEE ICRA. May 2016.

[2] J. Qian et al. "The Design and Development of an Omni-Directional Mobile Robot Oriented to an Intelligent Manufacturing System.” In: Sensors (Basel) (Sept. 2017).

[3] Tătar et al. "Structures of the Omnidirectional Robots with Swedish Wheels". In: Mechatronic Systems and Materials IV. Vol. 198. May 2013, pp. 132-137.

[4] S. L. Dickerson and B. D. Lapin. "Control of an omnidirectional robotic vehicle with Mecanum wheels". In: NTC '91 - National Telesystems Conference Proceedings. Mar. 1991, pp. 323-328.

[5] K. Kanjanawanishkul. "Omnidirectional wheeled mobile robots: wheel types and practical applications". In: International Journal of Advanced Mechatronic Systems. Vol. 6. Feb. 2016.

[6] R. Isermann and P. Ballé. "Trends in the Application of Model Based Fault Detection and Diagnosis of Technical Processes". In: IFAC Proceedings Volumes. Vol. 29. June 1996.

[7] Janos Gertler. "Fault Detection and Diagnosis". In: Encyclopedia of Systems and Control (2014). DOI: DOI10.1007/978-1-4471-5102-9_223-1.
[8] R. Isermann. "Supervision, fault-detection and faultdiagnosis methods, an introduction". In: Control engineering practice, 5(5), 639-652, 1997.

[9] J. Chen and R.J. Patton. Robust Model-Based Fault Diagnosis for Dynamic Systems. The International Series on Asian Studies in Computer and Information Science. Springer US, 1998.

[10] C. Zixing D. Zhuohua and Y. Jinxia. "Fault Diagnosis and Fault Tolerant Control for Wheeled Mobile Robots under Unknown Environments: A Survey". In: Proceedings of IEEE ICRA. 2005.

[11] K. Anastassia and P. Plöger. "Model-Based Fault Diagnosis Techniques for Mobile Robots". In: IFACPapersOnLine 49 (2016).

[12] S. I. Roumeliotis, G. S. Sukhatme, and G. A. Bekey. "Sensor fault detection and identification in a mobile robot”. In: Proceedings. IEEE/RSJ ICIRS . Innovations in Theory, Practice and Applications. Oct. 1998.

[13] L. Yutian and C. Jungan. "Integrated Fault Diagnosis Method of Mobile Robot". In: Communications in Computer and Information Science. 164 (2011).

[14] G.K. Fourlas, G.C. Karras, and K.J. Kyriakopoulos. "Sensors fault diagnosis in autonomous mobile robots using observer Based technique”. In: ICRA. May 2015.

[15] G.K. Fourlas et al. "Model based actuator fault diagnosis for a mobile robot". In: IEEE International Conference on Industrial Technology (ICIT). Feb. 2014.

[16] S. Mellah et al. "On fault detection and isolation applied on unicycle mobile robot sensors and actuators". In: 7th International Conference on Systems and Control (ICSC). IEEE. 2018, pp. 148-153.

[17] S. Mellah et al. "Detection isolation of sensor and actuator additive faults in a 4-mecanum wheeled mobile robot (4-MWMR)". In: International Conference on Control, Automation and Diagnosis (ICCAD). 2019.

[18] Dalil Ichalal et al. "Unknown input observer for LPV systems with parameter varying output equation". In: IFAC-PapersOnLine 48.21 (2015), pp. 1030-1035.

[19] S.R. Sahoo, S.S. Chiddarwar, and V. Alakshendra. "Intuitive dynamic modeling and flatness-based nonlinear control of a mobile robot”. In: SIMULATION (2018).

[20] Alberto Isidori and Claudio De Persis. "Feedback linearization of nonlinear systems". In: Control Systems, Robotics and AutomatioN-Volume XII: Nonlinear, Distributed, and Time Delay Systems-I (2009).

[21] Jie Chen and Ron J Patton. Robust model-based fault diagnosis for dynamic systems. Vol. 3. Springer Science \& Business Media, 1999.

[22] Paul M Frank. "Fault diagnosis in dynamic systems via state estimation-a survey". In: System fault diagnostics, reliability and related knowledge-based approaches. Springer, 1987, pp. 35-98.

[23] R.N. Clark. "Instrument fault detection". In: IEEE Trans. Aerospace and Electronic Systems. 1978, pp. 456-465.

[24] F. Pukelsheim. "The Three Sigma Rule". In: The American Statistician (1994). 